\title{
Sugar export limits size of conifer needles
}

\author{
Rademaker, Hanna; Zwieniecki, Maciej A.; Bohr, Tomas; Jensen, Kaare Hartvig
}

Published in:

Physical Review E

Link to article, DOI:

10.1103/PhysRevE.95.042402

Publication date:

2017

Document Version

Publisher's PDF, also known as Version of record

Link back to DTU Orbit

Citation (APA):

Rademaker, H., Zwieniecki, M. A., Bohr, T., \& Jensen, K. H. (2017). Sugar export limits size of conifer needles. Physical Review E, 95(4), 042402. https://doi.org/10.1103/PhysRevE.95.042402

\section{General rights}

Copyright and moral rights for the publications made accessible in the public portal are retained by the authors and/or other copyright owners and it is a condition of accessing publications that users recognise and abide by the legal requirements associated with these rights.

- Users may download and print one copy of any publication from the public portal for the purpose of private study or research.

- You may not further distribute the material or use it for any profit-making activity or commercial gain

- You may freely distribute the URL identifying the publication in the public portal

If you believe that this document breaches copyright please contact us providing details, and we will remove access to the work immediately and investigate your claim. 


\title{
go \\ Sugar export limits size of conifer needles
}

\author{
Hanna Rademaker, ${ }^{1}$ Maciej A. Zwieniecki, ${ }^{2}$ Tomas Bohr, ${ }^{1}$ and Kaare H. Jensen ${ }^{1, *}$ \\ ${ }^{1}$ Department of Physics, Technical University of Denmark, DK 2800 Kgs. Lyngby, Denmark \\ ${ }^{2}$ Department of Plant Sciences, University of California at Davis, Davis, California 95616, USA
}

(Received 5 January 2017; revised manuscript received 10 February 2017; published 7 April 2017)

\begin{abstract}
Plant leaf size varies by more than three orders of magnitude, from a few millimeters to over one meter. Conifer leaves, however, are relatively short and the majority of needles are no longer than $6 \mathrm{~cm}$. The reason for the strong confinement of the trait-space is unknown. We show that sugars produced near the tip of long needles cannot be exported efficiently, because the pressure required to drive vascular flow would exceed the greatest available pressure (the osmotic pressure). This basic constraint leads to the formation of an inactive region of stagnant fluid near the needle tip, which does not contribute to sugar flow. Remarkably, we find that the size of the active part does not scale with needle length. We predict a single maximum needle size of $5 \mathrm{~cm}$, in accord with data from 519 conifer species. This could help rationalize the recent observation that conifers have significantly smaller leaves than angiosperms, and provide a biophysical explanation for this intriguing difference between the two largest groups of plants.
\end{abstract}

DOI: 10.1103/PhysRevE.95.042402

\section{INTRODUCTION}

Light capture and gas exchange associated with photosynthesis take place in plant leaves, organs strikingly adapted for these processes that are essential to life on Earth. A wide range of morphological and physiological traits are expressed in plant leaves. This has been appreciated since antiquity [1], but recent studies have revealed that only a remarkably small portion of the available trait-space is occupied by extant species [2,3]. The physical mechanisms that impose constraints on the traits favored by evolution, however, remain poorly understood. A particularly intriguing case is that of needle or scale-bearing gymnosperms (conifers). Most species $(75 \%)$ in this group have leaves that are shorter than $6 \mathrm{~cm}$ in length; only one genus (Pinus) has multiple species with needles longer than $25 \mathrm{~cm}$. By contrast, leaves on broad-leaved angiosperms grow much larger. The mechanisms that limit the size of conifer needles are unknown.

Plant leaves are typically thin and flat, thereby maximizing the surface area exposed to light and promoting photosynthesis in which light energy is converted into chemical energy stored in sugar molecules. Leaves are generally arranged on the plant so as to expose their surfaces to light with little self-shading. Leaf size and shape is influenced by a number of factors, including light interception, gravity, wind, temperatures, herbivores, and vascular transport efficiency [4,5]. The greatest diversity is found among broad-leaved angiosperm species, where leaf diameter spans three orders

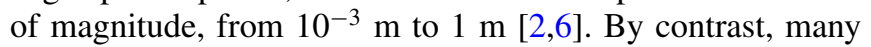
gymnosperm species have slender needle- or scale-like leaves, which are significantly smaller. Although needles and scales are thought to be advantageous in dry or cold climates frequented by snow and frost, the mechanistic explanation why their size is constrained is missing [2].

The vascular architecture of leaves may hold clues to the reason for the difference in size between angiosperm and gymnosperm leaves. It is well established that a major

\footnotetext{
*khjensen@fysik.dtu.dk
}

innovation in angiosperms concerns xylem vessels, the vascular conduits that deliver water to leaves actively used in photosynthesis and passively lost to the atmosphere while obtaining $\mathrm{CO}_{2}$. Angiosperm vessels are longer and largerdiameter conduits than gymnosperm tracheids, permitting higher hydraulic conductivities. This, together with a greater density of leaf veins, has made it possible for angiosperms to extend the range of leaf photosynthetic capacities to higher values and presumably also contributed to the evolution of large leaves in angiosperms [7].

However, the situation is remarkably different for the phloem vascular conduits, which transport sugars produced by photosynthesis between leaves and sites of growth or storage (e.g., roots, fruits, and shoots). In the stem, the cross sectional area $A$ of phloem conduits is approximately equal in angiosperms and gymnosperms $\left(A_{\text {stem }} \sim 500-1000 \mu \mathrm{m}^{2}\right.$; see Ref. [8]). In contrast, phloem conduits in conifer leaves are remarkably small, with areas in the range $A \sim 3-10 \mu \mathrm{m}^{2}$ [9]. While the factors that limit phloem cell size to this range in conifer leaves are unknown, it is clear that the length and size of vascular conduits must impose constraints on sugar export rates. The pressure differential $\Delta p$ required to drive flow over a length $L$ scales inversely with the conduit area $A$, as $\Delta p \sim u L \eta / A$, where $u$ is the flow speed and $\eta$ is the sap viscosity. Thus a long and narrow tube requires a relatively large pressure $\Delta p$ to sustain similar sugar export. However, because the sap flow is driven by osmosis, the available pressure is always limited by the cell's osmotic pressure, i.e., $\Delta p \leqslant R T c$, where $R$ is the gas constant, $T$ is temperature and $c$ is sugar concentration. This is likely to reduce the efficiency of transport in relatively long needles.

Nevertheless, it should be noted that gymnosperms dominated the Earth from around 300 million years ago (mya) until the evolution and diversification of angiosperms ( 120 mya) and still have an advantage over angiosperms in extreme environments. Moreover, conifer trees hold the records for the world's tallest (112.7 m, Sequoia sempervirens) [10], widest (14.5 m, Taxodium mucronatum) [11], oldest ( $\sim 900 \mathrm{yr}$, Pinus longaeva) [12] and largest (1486 $\mathrm{m}^{3}$, Sequoiadendron giganteum) [13] trees. These numbers suggest that although 
various factors may limit the absolute size of conifer leaves, the organisms are not suffering from small leaf size and we can expect photosynthesis and sugar export from conifer leaves to be highly efficient.

To explore the organization of flows within needles, and to rationalize the limits to their size, we proceed in the following way. First, we present data which demonstrate that conifer leaf lengths occupy a narrow range of sizes. Then we develop a mathematical model for sugar export from a slender leaf. Next, we explore the dependence of the sugar export rate on the geometric and material parameters. We show that a characteristic needle length exists, above which sugar export becomes inefficient due to the formation of a region of stagnant fluid near the needle tip. Finally, we conclude and discuss the implications for our understanding of observed leaf lengths in nature and related physiological transport problems.

\section{RESULTS}

\section{A. Conifer leaf size}

A wide range of morphological and physiological traits are expressed in plants. To our knowledge, the diversity in leaf size and function was first discussed systematically by Theophrastus around $300 \mathrm{BC}$, who noted that "To sum up, the differences between leaves are shewn in size, number, shape, hollowness, in breadth, roughness. ... These are all the differences in leaves stated somewhat generally, and this is a fairly complete list of examples" ([1], p. 77). This classic and countless subsequent works have contributed to our understanding of the factors that influence leaf size and shape [4,14]. Diaz et al., however, recently reported that while diversity may appear almost unbounded, only a relatively small portion of the available trait space is occupied by extant species [2]. Only particular ranges of traits - for instance, plant height, stem density, seed mass, and leaf size-are found in nature. The reason for this confinement remains elusive, and Diaz et al. state that "Our results are correlative and cannot prove rigorously why such a large share of the potential trait volume is not occupied. Still, from first principles many more combinations of traits than those observed seem feasible as far as biomechanics and evolutionary genetics are concerned" ([2], p. 170).

The greatest diversity in leaf size is found among broadleaved angiosperm woody species, where leaf diameter spans three orders of magnitude, from $1 \mathrm{~mm}$ to over $1 \mathrm{~m}$ [6]. The median leaf length from over 1900 species representing 95 families was $18 \mathrm{~cm}$ with $97 \%$ of species with leaves longer than $6 \mathrm{~cm}$ [6].

By contrast, we find that conifers-which have slender needle- or scale-like leaves-are significantly smaller. The data plotted in Fig. 1 represent leaf size from 519 species in six families and were obtained from The Gymnosperm Database [15]. All values and source references are available in the supporting online material (Table S1) [16]. With few exceptions, a typical maximal and minimal value of leaf length is given in The Gymnosperm Database. In Fig. 1 we plotted the mean of these two values, with the horizontal line indicating the full range. For 37 species only one typical value was given in the database, in which case we used this value instead of

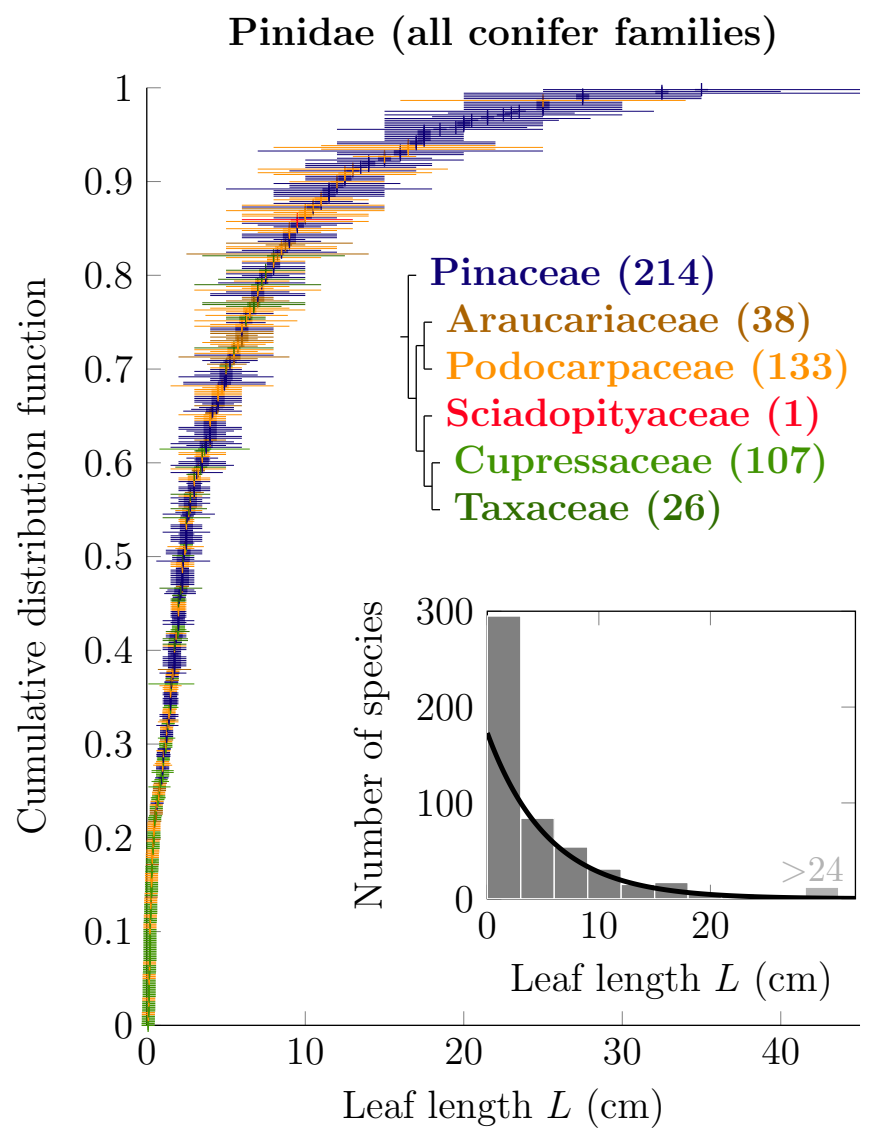

FIG. 1. Conifer leaf size follows a narrow distribution with a high density of short leaves. Cumulative distribution function (cdf) of leaf length is plotted as a function of the leaf length $L$ for 519 species (six families). Species are sorted according to their mean leaf length, and the horizontal lines indicate the observed range of leaf sizes for each species. The median leaf length is $2.3 \mathrm{~cm}, 75 \%$ of species have leaves shorter than $6 \mathrm{~cm}$, while $97.5 \%$ have leaves no longer than $23 \mathrm{~cm}$. Inset shows a histogram of the mean leaf length divided into bins of $3 \mathrm{~cm}$. Note that leaf length is approximately exponentially distributed (solid line shows exponential fit as a guide to the eye). Data and source references are available in the supporting online material, Table S1 [16].

the mean. The leaf length $L$ has median value $2.3 \mathrm{~cm}, 75 \%$ of species have leaves shorter than $6 \mathrm{~cm}$, and $97.5 \%$ have leaves no longer than $23 \mathrm{~cm}$. Moreover, leaf length follows an approximately exponential distribution characterized by a high density of species at short leaf lengths. As stated previously, the reason for the clustering of sizes in the range below $6 \mathrm{~cm}$ is unknown.

\section{B. Efficiency of sugar export}

To explore the biomechanics of sugar export from slender leaves, and to rationalize the limits to their size, we proceed by considering a mathematical model for sugar transport in conifer needles. Our approach follows previous models applied to describe aspects of long-distance sugar transport in plants [17-20], suction-driven flow in roots [21] and leaves [22], and transport in long narrow epithelia channels which absorb or secrete fluids [23,24]. 
Plant leaves capture energy from the sun and store it in the chemical bonds of sugars. These energy-rich molecules are distributed to distal parts of the plant and used in processes essential to cellular metabolism, organism growth, and reproduction. Sugar transport takes place in phloem cells which form a continuous microfluidic channel network from leaf to root. The current hypothesis for phloem transport dates to the 1920s when Ernst Münch proposed that gradients in cell turgor pressure drive the bulk flow of sugars and other solutes from sources to sinks [25]. Sources are regions of the plant where photosynthesis (or the breakdown of starch) results in high concentrations of sugars and thus large turgor pressures due to osmosis. In contrast, sinks are regions of the plant where mitochondrial respiration and the incorporation of sugars into larger molecules reduces the concentration of osmotically active solutes and thus lowers turgor pressures. According to the Münch hypothesis, transport through the phloem results from these osmotically generated differences in pressure and occurs without any additional input of energy. In needles, phloem tubes are situated inside vascular bundles [Figs. 2(a)-2(b)]. Most of the 5-50 bundled tubes that run in parallel span the entire length of the needle [26]. We therefore treat the dynamics of phloem transport in each conduit separately, assuming no interactions or exchange of material between adjacent channels. Our goal in the following is to quantify how quickly sugars can be removed (exported) from a needle, and how the efficiency of the process depends on the size of the needle and of the phloem conduits.

Sugar molecules are produced in the mesophyll by photosynthesis and subsequently diffuse into the phloem conduits. To quantify how quickly they can be exported, we consider a simple model of osmotic transport in a microchannel [Fig. 2(c)]. The presence of sugar molecules in the phloem cells leads to an osmotic flux of water $J=L_{p}(R T c-p)$ across the cell membrane, where $c$ is the concentration of sugar, $R$ is the gas constant, $T$ is temperature, $p$ is cell turgor pressure, and $L_{p}$ is the membrane permeability. Due to the uptake of water, the flow velocity $u(x)$ inside the conduit builds up along the $x$-axis from the tip of the needle at $x=0$ [where $u(0)=0$ ], as

$$
\frac{d u(x)}{d x}=\frac{2 L_{p}}{r}[R T c(x)-p(x)],
$$

where the parameter $r$ is the conduit radius [27]. Sieve tubes in needles are relatively small, with conductive areas in the range $A=3-10 \mu \mathrm{m}^{2}$ [9] [Fig. 2(d)]. These export conduits are quite narrow when compared to tube sizes observed in leaves of flowering plants (e.g., $A=16.2-89.5 \mu \mathrm{m}^{2}$ and $A=$ $12.9-29.9 \mu \mathrm{m}^{2}$ in maize and barley $\left.[28,29]\right)$ and also in stems of both gymnosperms and angiosperms $\left(A \sim 500-1000 \mu \mathrm{m}^{2}\right.$ [6]). Conservation of sugar mass leads to the continuity equation

$$
\frac{d}{d x}(u c)=\gamma,
$$

where $\gamma$ is the amount of sugar loaded from the mesophyll into the phloem per unit volume and time. The sugar export flux from the needle - and its dependence on physical parameters - can be determined from a solution to (1) and (2) with appropriate boundary conditions. This will allow us to ascertain the efficiency of the process under various conditions. (a)
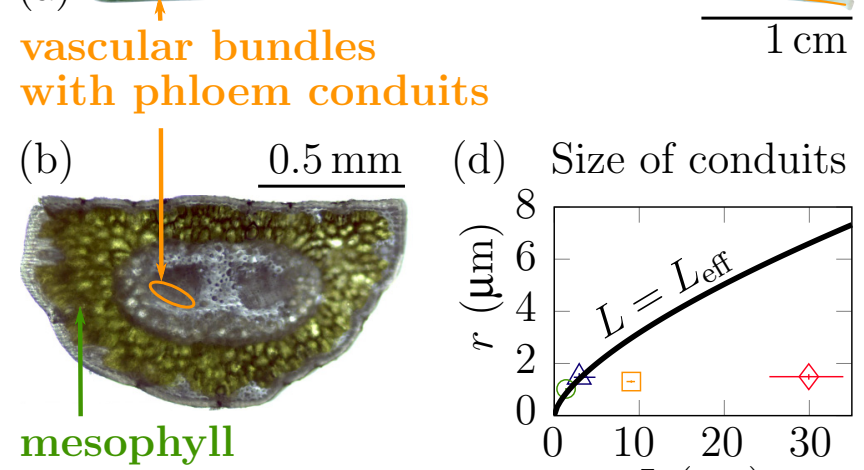

(d) Size of conduits
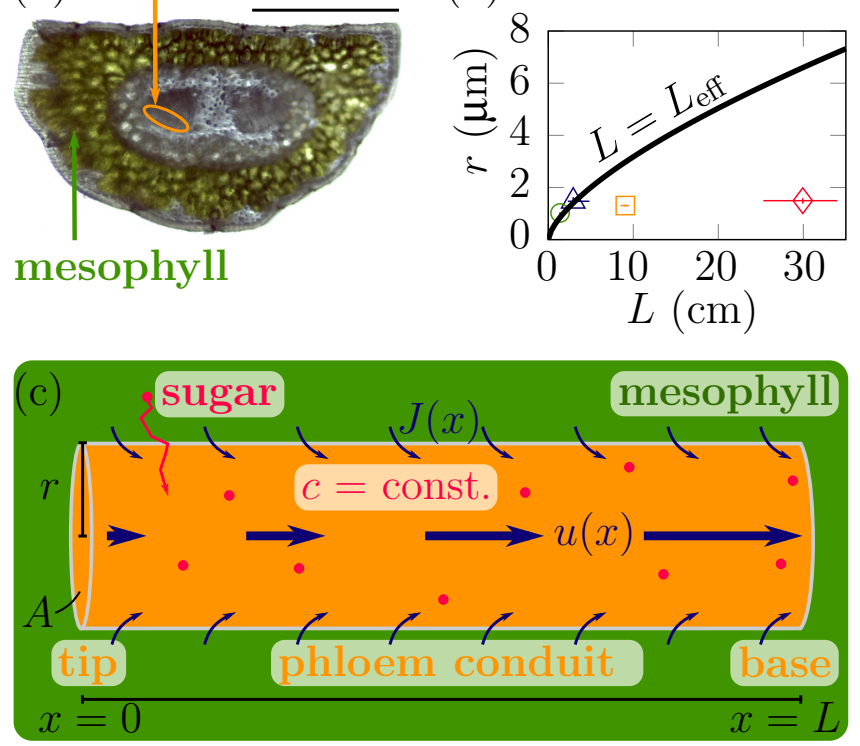

FIG. 2. Biomechanics of osmotic sugar export. (a, b) Conifer needles are long and slender leaves which capture sunlight to photosynthesize sugars in the mesophyll cells (green tissue). Microfluidic phloem vascular conduits facilitate export of sugars to distal parts of the plant through a file of cellular tubes (sieve elements) located near the central axis of the needle (orange tissue). (c) We model a file of sieve elements as a single circular microchannel with radius $r$ and length $L$. Sugars diffuse from the mesophyll into the phloem, and export is driven by osmotic uptake of water over the surface of the phloem at a flux $J=L_{p}(R T c-p)$, where $c$ is the phloem sugar concentration, $p$ is the phloem pressure, and $L_{p}$ is the permeability of the cell membrane. The water potential outside the phloem is assumed to be zero $\left(R T c_{\mathrm{ext}}-p_{\mathrm{ext}}=0\right)$. The velocity inside the tube increases from $u(0)=0$ to $u(L)$ according to (1). (d) Phloem tube radii are remarkably uniform among species with leaf length in the range $2-30 \mathrm{~cm}$. Plot showing mean individual conduit radius $r$ as a function of needle length $L$ for Picea omorika (green circle), Abies nordmanniana (blue triangle), Pinus cembra (orange square), and Pinus palustris (red diamond). Error bars indicate maximum error. Data reproduced from Ref. [9]. The black line indicates the predicted conduit radius from our model assuming $L=L_{\text {eff }}$ [Eq. (8)].

In the following, we therefore seek to compute the total sugar flux out of the needle $\Gamma=u(L) c(L)$, where the position $x=L$ corresponds to the needle base. We also determine the local loading rate $\gamma(x)$, which measures how much each part of the needle contributes to the production and export of energetic sugars. Finally, we determine the conduit pressure $p(x)$.

Before proceeding, however, we make two simplifying assumptions. First, we assume that the pressure variation and the flow velocity along the conduit are related by Darcy's law, $d p / d x=-8 \eta u(x) / r^{2}$, with $\eta \simeq 4 \mathrm{mPas}$ the viscosity of the fluid [30]. This is reasonable because the channel aspect ratio $r / L \sim 10^{-4}$ is small, and hence the lubrication approximation is valid. Second, we assume that the sugar concentration 
$c(x)=c$ is constant inside the conduit. Sugar molecules produced in the mesophyll are loaded into the phloem conduits by either protein pumps or by bulk flow and diffusion through plasmodesmata nanopores [31]. Evidence suggests that plasmodesmata transport is the dominant mechanism in needles [32]. Our analysis, however, does not depend on details of the loading mechanism, as long as it is able to preserve a constant concentration inside the conduit. Presuming that diffusion is the slowest of the relevant transport processes, the assumption $c=$ const therefore at most requires that sugar molecules which are advected by the bulk flow along the needle can be quickly replenished by radial diffusion, i.e., that the Peclet number Pe $=u r / D<1$. Using typical values $\left(u=5 \times 10^{-5} \mathrm{~m} / \mathrm{s}, r=10^{-6} \mathrm{~m}\right.$ and $\left.D=5 \times 10^{-10} \mathrm{~m}^{2} / \mathrm{s}\right)$ we find $\mathrm{Pe}=0.1$ in accord with the assumption $c(x)=$ const.

With these simplifications, the governing equation (1) becomes

$$
\frac{d^{2} u(x)}{d x^{2}}=16 \frac{L_{p} \eta}{r^{3}} u(x) .
$$

The solution for the flow velocity $u(x)$ with boundary condition $u(0)=0$ is

$$
u(x)=\frac{1}{2} \sqrt{\frac{r L_{p}}{\eta}} \frac{\sinh \left(\sqrt{M} \frac{x}{L}\right)}{\cosh \sqrt{M}}[R T c-p(L)],
$$

where $p(L)$ is the pressure at the needle base, and the nondimensional Münch number $M=16 \eta L_{p} L^{2} / r^{3}$ characterizes the relative importance of viscous and membrane flow resistances. The outlet pressure $p(L)$ can be determined from the sink (e.g., root) pressure $p_{\text {sink }}$ and the hydraulic resistance of the stem $R_{\text {stem. }}$. This leads to $p(L)-p_{\text {sink }}=Q R_{\text {stem }}=$ $\pi r^{2} u(L) R_{\text {stem }}$. An explicit expression for the pressure can be obtained from (4), which leads to

$$
R T c-p(L)=\frac{R T c}{1+\pi r^{2} R_{\text {stem }} \frac{1}{2} \sqrt{\frac{r L_{p}}{\eta}} \tanh \sqrt{M}},
$$

where we have assumed water potential equilibrium at the sink $p_{\text {sink }}=0$. We note that (4)-(5) are in accord with Eq. (1) of Ref. [33] when $M \ll 1$, i.e., for relatively short leaves. From the expression in (4) we can compute the loading rate $\gamma(x)=c u^{\prime}$ and pressure $p(x)$ from (1):

$$
\begin{gathered}
\gamma(x)=\frac{2 L_{p}}{r} c \frac{\cosh \left(\sqrt{M} \frac{x}{L}\right)}{\cosh \sqrt{M}}[R T c-p(L)], \\
p(x)=R T c-\frac{\cosh \left(\sqrt{M} \frac{x}{L}\right)}{\cosh \sqrt{M}}[R T c-p(L)] .
\end{gathered}
$$

For small values of $M$ (corresponding to, say, a short and wide tube), the velocity $u$ is a linear function of $x$, pressure decreases gradually, and the loading rate $\gamma=c u^{\prime}$ is constant throughout the needle (Fig. 3). Each part of the needle thus contributes equally to the sugar export. In contrast, the situation is completely different when $M$ is large, relevant for example in a long and narrow tube. In this case, the velocity profile is strongly nonlinear and characterized by a stagnant zone at the needle tip. Near the base of the needle the speed $u$ and loading rate $\gamma$ both grow exponentially with position; $u, \gamma \propto \exp [\sqrt{M}(x / L-1)]$, hence most material is collected from this region. Close to the tip the speed is relatively slow and almost no loading occurs [i.e., $\gamma(x) / \gamma(L) \ll 1$ and
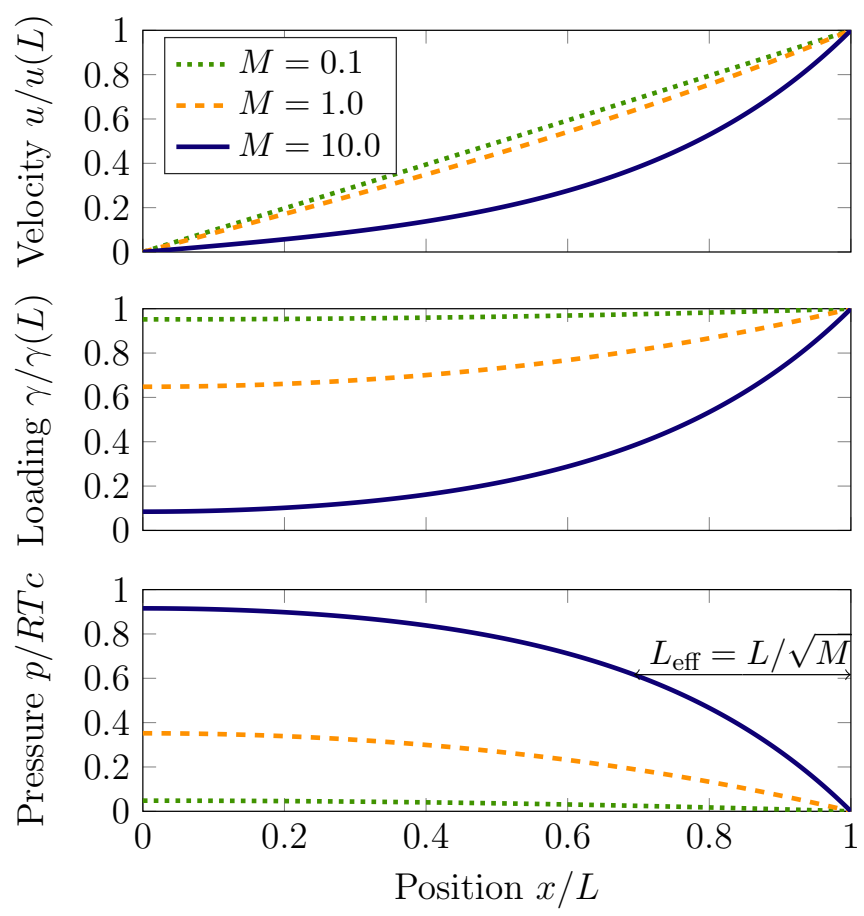

FIG. 3. Very long conduits are inefficient. Velocity $u$, loading $\gamma$, and pressure $p$ plotted as a function of relative position $x / L$, as calculated from Eqs. (4) to (7), assuming $p(L)=0$. For small Münch numbers $M$ (e.g., short and wide conduits) the velocity $u$ increases linearly along the tube and the loading function $\gamma=c d u / d x$ is approximately constant. For large $M$ (e.g., long and narrow conduits) a stagnant zone forms near the tip $(x=0)$, and gradients in velocity, loading, and pressure are localized near the needle base. The main contribution to flow and sugar export comes from a region of length $L_{\text {eff }}=L / \sqrt{M}$ adjacent to the needle base [(8), indicated by an arrow for $M=10]$. Velocity and loading are normalized with respect to their terminal values $(u(L), \gamma(L))$ while pressure is plotted relative to the maximum value set by the osmotic pressure $R T c$. The Münch numbers corresponding to data from the four conifer species displayed in Fig. 2(d) are $M \approx 1$ (A. nordmanniana and $P$. omorika), $M \approx 10$ (P. cembra), and $M \approx 100$ (P. palustris).

$u(x) / u(L) \ll 1]$. Likewise, the pressure gradient is localized close to the needle base. In the limit of large $M$, the contribution from each part of the needle thus varies strongly, and the most significant contribution comes from a region closest to the needle base. The size of this region is the intrinsic length scale of the exponential,

$$
L_{\mathrm{eff}}=\frac{L}{\sqrt{M}}=\frac{r^{3 / 2}}{\left(16 L_{p} \eta\right)^{1 / 2}},
$$

where we used the definition of the Münch number. Remarkably, this effective needle length $L_{\text {eff }}$ is independent of needle length $L$ as well as the sugar concentration $c$. We note that related models have been analyzed in the context of long-distance sugar transport in plants [17,18] and to model transport in epithelia channels [23,24]. These systems, however, are characterized by a separation of scales, where material is added to the channel in a relatively short active portion of the channel of known length. In contrast, material is added along the entire channel in our system, and the size 


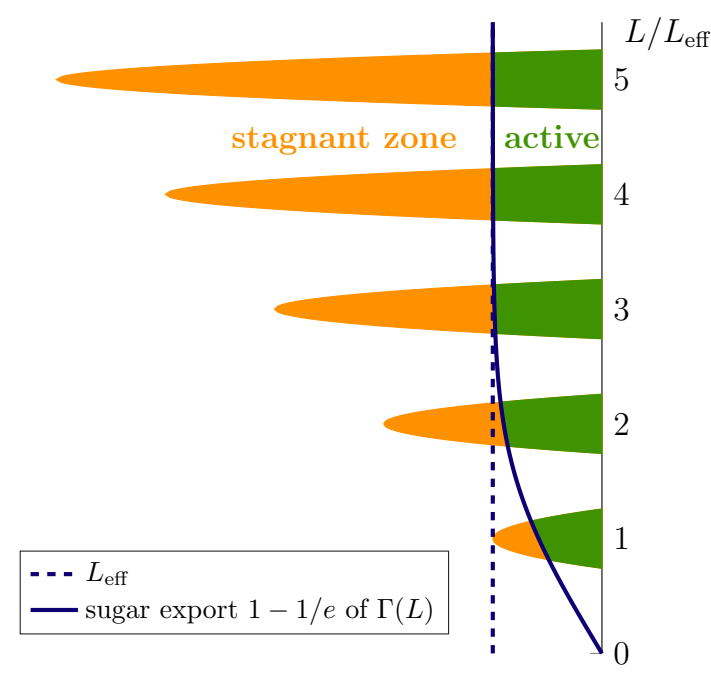

FIG. 4. Sugars exported from conifer needles originate predominantly from an active region of length $L_{\mathrm{eff}}=r^{3 / 2} /\left(16 \eta L_{p}\right)^{1 / 2}$ near the base [shaded green area, (8)]. This region contributes $63 \%(=1-1 / e)$ to the total sugar export $\Gamma(L)$. The dashed vertical line indicates $L=L_{\text {eff }}$.

of the active region $L_{\text {eff }}$ is an emergent property of the system. Moreover, we note that the active part of the channel is towards the needle base (Fig. 4), and not towards the tip as assumed in Segel's standing gradient problem [23]. It is interesting that the expression for the effective length is independent of the concentration $c$. In fact, one can take $c=0$ and drive the fluid through the porous walls by making the pressure inside the tube smaller that the external one. This is what happens in the xylem of plant roots, where suction-driven flow facilitated by leaf transpiration leads to similar phenomena [21].

The total sugar flux leaving the needle $\Gamma=c u(L)$ allows us to gauge the relative performance of different needle designs as a function of the system parameters [using (4), (8), and the definition of the Münch number]:

$$
\Gamma=c u(L)=\frac{1}{2} \sqrt{\frac{r L_{p}}{\eta}} c \tanh \left(\frac{L}{L_{\mathrm{eff}}}\right)[R T c-p(L)] .
$$

From (9) we again find that the effective length $L_{\text {eff }}$ is the characteristic size above which sugar export no longer scales linearly with needle length and very little can be gained by a further increase in length (Fig. 4). For instance, increasing needle size to $10 L_{\text {eff }}$ only increases sugar export by a factor 1.3.

The existence of a stagnant zone for lengths larger than $L_{\text {eff }}$ is not restricted to the flow in a single tube with a constant concentration. In fact, one can show [34] that a system of coupled tubes will behave in essentially the same way, although the details depend on exactly how the tubes are distributed. Modeling the phloem of the needles as a stack of coupled tubes $[9,22]$, distributed as a power law such that

$$
N(x)=N_{0}\left(\frac{x}{L}\right)^{a}
$$

is the number of sieve tubes at distance $x$ from the tip, one finds expressions for the velocity and the flow rate, again using the constant concentration approximation [34], which are completely analogous to the single tube results given above, when the factor $\sinh \left(\sqrt{M} \frac{x}{L}\right) / \cosh \sqrt{M}$ appearing in (4) is replaced by $\left(\frac{x}{L}\right)^{b} I_{b}\left(\sqrt{M} \frac{x}{L}\right) / I_{b-1}(\sqrt{M})$ where $b=(a+1) / 2$ and $I_{b}$ is the modified Bessel function of order $b$. The modified Bessel functions are very analogous to the hyperbolic functions found for a single tube, and again the characteristic length is $L_{\text {eff }}=L / \sqrt{M}$.

The assumption of constant sugar concentration is essential for deriving the analytical results presented so far. This is an approximation which has been made before (e.g., in Ref. [33]). In Ref. [9] a different approximation is made: that of constant loading. For small needles the result is the same. As seen in Fig. 3(b), constant concentration does lead to a roughly constant loading rate as long as $L \sim L_{\text {eff. When }}$ $L \gg L_{\text {eff, }}$ which is rare in needles according to our results, the constant loading assumption is untenable, since the pressures near the tip of the needle would build up to unrealistic heights. As mentioned above, we assume that the available pressure is always limited by the cell's osmotic pressure, i.e., $\Delta p \leqslant R T c_{0}$, where $c_{0}$ is the largest feasible concentration. Moreover it turns out that the case of constant concentration used here gives the maximal possible sugar export: If we assume that the plants can only generate concentrations up to some maximal value $c_{0}$, then the flow with $c(x)=c_{0}$ everywhere will have the largest velocity (see Appendix), and therefore the largest sugar export $\Gamma(L)=c(L) u(L)$. Obviously, it would be very interesting to know how well the assumption of constant concentration describes real needles.

We end our analysis of sugar export by briefly discussing the physical reason for the existence of the effective length (8) and its dependence on system parameters. The pressure gradient generated by the flow is set by viscous Darcy friction $d p / d x \sim u \eta / r^{2}$, but the maximum pressure attainable is the osmotic pressure $R T c$. This means that the pump can only sustain a flow speed $u$ over a distance $\ell$ which obeys $R T c / \ell \sim u \eta / r^{2}$. The velocity itself, however, also depends on the factor $R T c$ through (1), hence its maximum value scales as $u \sim L_{p} R T c \ell / r$. This sets an upper limit to the pipe length which can carry osmotic flow set by the scaling law $\ell \sim r^{3 / 2} /\left(\eta L_{p}\right)^{1 / 2}$, in accord with (8). It is quite surprising that the magnitude of the effective length does not depend on the available pressure differential $\Delta p=R T c$ (and hence sugar concentration $c$ ). This appears to be an intrinsic property of the osmotic flow process, where the Darcy pressure gradient is the result —and not cause - of the liquid flow.

In summary, we have shown that sugar export is influenced by a number of factors, including needle length. We have determined that a characteristic needle size exists which controls the efficiency of sugar export, and we have shown that if the needle length $L$ exceeds $L_{\text {eff }}$, sugar output does not increase significantly. If these results are applicable to sugar export from conifer needles, we expect to find that $L_{\text {eff }}$ provides an upper estimate of their length. This hypothesis is tested below.

\section{DISCUSSION}

A relatively complete picture of the factors that influence sugar export from conifer needles and similar slender leaves 


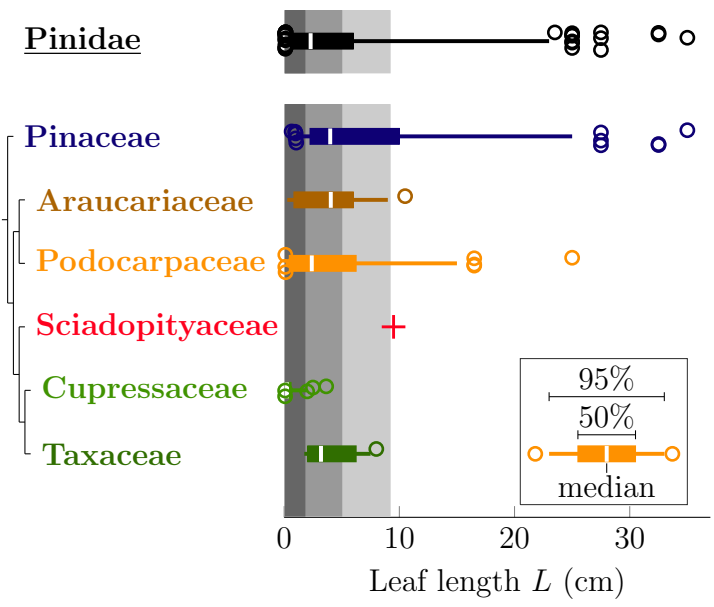

FIG. 5. Efficient leaves are found in multiple different conifer groups. Box and whisker graph of the phylogenetic distribution of mean leaf length $L$ across extant conifers. Shaded gray regions indicate maximum leaf length predicted by (8) for phloem conduit radii $r=1,2,3 \mu \mathrm{m}$. The vertical line indicates the distribution median, while $50 \%$ of the data points are inside the box and 95\% are inside the whiskers. Open circles indicate outliers. The Sciadopityaceae family, marked with a single "+", consists of only one species. Data and source references are available in the supporting online material, Table S1 [16].

has emerged. First and foremost, we have found that the osmotic flow mechanism used to export sugars imposes fundamental limitations on leaf size. For leaves longer than $L_{\text {eff }}=r^{3 / 2} /\left(16 L_{p} \eta\right)^{1 / 2}$, transport efficiency is compromised by the formation of a region of stagnant fluid [(8); Fig. 4]. The vascular conduits in the stagnant region cannot contribute to the export of sugars from the leaf, because the pressure required to do so would exceed the osmotic pressure. We note that the intrinsic active length scale $L_{\text {eff }}$ is not expected to emerge from an analysis of the related standing gradient flow problem [23,24], because that analysis assumes loading occurs in a known, relatively short, region of the channel.

The diversity of leaf sizes found in nature provides an ideal opportunity to test the generality of the results demonstrated by our analysis. When compared to the prediction that the effective leaf length $L_{\text {eff }}$ provides an upper limit to leaf length [see (8)], our theory gives an upper bound $L_{\text {eff }}=5.0 \mathrm{~cm}$, obtained with $r=2 \mu \mathrm{m}, L_{p} \sim 5 \times 10^{-14} \mathrm{~m} / \mathrm{s} / \mathrm{Pa}$, and $\eta=4$ mPas [8,17]. We note that the effective length $L_{\text {eff }} \sim r^{3 / 2}$ is sensitive to variation in conduit radius $r$. The range of observed values is $r=1 \mu \mathrm{m}$ to $3 \mu \mathrm{m}$ corresponding to effective lengths between $L_{\text {eff }}=1.8 \mathrm{~cm}$ and $L_{\text {eff }}=9.2 \mathrm{~cm}$. The predicted $L_{\text {eff }}$ correlates with the median length of conifer needles (Fig. 5). Moreover, the observed needle length in species where the conduit dimensions are known is in reasonable accord with theoretical predictions [Fig. 2(d)], with the notable exception of Pinus palustris, which is significantly longer than predicted (see discussion below).

From an evolutionary perspective, we note that conifer leaves most likely evolved once and are homologous in different conifer families (Fig. 5). Thus it might not be surprising that despite conifers covering a wide range of geographic and environmental conditions [35], their leaves remain highly constrained in length, presumably due to the small radius of phloem tubes. Such a constraint results in an effective limit on sugar export rate from a single leaf, and if high productivity and competitive growth rates of trees have to be achieved, it can only be realized by an increased leaf number per stem length, as observed in conifers [36-38].

Our results could thus help rationalize the recent observation that conifers have significantly smaller leaves than angiosperms [2] and provide a biophysical explanation for this intriguing difference between the two largest groups of plants. Several exceptions to the rule are found in the genus Pinus, which has a number of species with needle lengths exceeding $L_{\text {eff }}$, suggesting the presence of other trade-offs associated with length than the stagnant-zone effect. This particular inefficiency might be an adaptive trait related to the evolutionary history of the genus. Although the genus Pinus evolved in the Cretaceous, major species radiation occurred with the evolution of grasses and grassland ecosystems in the Miocene [35,39]. Thus this radiation could be interpreted as a response to newly emerged competition. In the juvenile phase fast-growing, long needles would be best suited to compete with long leaf grasses, where the tip is the most productive part of the leaf. Once off the grass layer, needle length is reduced by a significant fraction, in the case of Pinus palustris by a factor $1 / 2$ from approximately $45 \mathrm{~cm}$ to $20 \mathrm{~cm}$. Though still representing significant deviation from $L_{\text {eff }}$, such a reduction suggests a developmentally limited but significant improvement of the needle's efficiency. We note that angiosperm leaf lengths in maize and barley (which are approximately linear in architecture) are consistent with predictions from (8); although the limited data set on phloem radius sizes does not allow us to draw definite conclusions [28,29], this suggests that the inefficiency of Pinus is only limited to this genus and not related to leaf shape.

Our analysis assumes a constant sugar concentration in the leaf phloem, a premise that could be tested using techniques developed to extract phloem sap from sieve elements [40,41]. Moreover, our model predicts patterns of sugar loading rates $\gamma$, which are a function of needle morphology (Fig. 3). The loading rate $\gamma$ depends on the local sugar production rate and on the rate of starch accumulation. Using a combination of leaf gas exchange system and starch analysis $[42,43]$ it appears technically feasible to test the prediction that loading rates are lower at the tip of long needles, when compared to rates at the base (see Figs. 3 and 4).

We end by emphasizing that efficient sugar export from leaves is one among many factors that allows the organisms to survive natural selection, physiological challenges, and competitive exclusions. Several environmental effects (e.g., wind, snow load, drought, light level) were previously used to explain limits to leaf sizes, their morphology, and the relative performance of species in particular environments $[4,44]$. Trade-offs are associated with each trait, and no single globally optimum strategy exists. Hence needles longer than $5 \mathrm{~cm}$ can exist, although we expect them to be rare. However, the intrinsic physiological properties role of transport has not previously been considered. We also note that our simplified 
model of sugar transport does not include details of several potentially important factors, including photosynthesis, sugar loading [32,45], and interactions between adjacent phloem conduits [9]. Consideration of these factors could improve prediction of size limits for particular species; nevertheless, the presented mechanistic model explaining upper bound to leaf size in conifers offers insight into the role of phloem physiology on morphology in conifers, and to our knowledge such an approach has not been found prior to this work.

\section{ACKNOWLEDGMENT}

H.R. was supported by the Danish Council for Independent Research | Natural Sciences (Grant No. 12-126055). K.H.J. was supported by a research grant (13166) from VILLUM FONDEN.

\section{APPENDIX}

We show in the following that the constant concentration profile $c(x)=c_{0}$ does indeed lead to the highest flow velocity at the end of the conduit $u(L)$, if $c_{0}$ is the maximum concentration the plant can generate. If we start again from (1) and assume any concentration profile $c(x)$, the governing equation is

$$
\begin{aligned}
\frac{d^{2} u(x)}{d x^{2}} & =\frac{2 L_{p}}{r}\left[R T \frac{d c(x)}{d x}+\frac{d p}{d x}\right] \\
& =\frac{2 L_{p} R T}{r} \frac{d c(x)}{d x}+\frac{M}{L^{2}} u(x),
\end{aligned}
$$

where we used Darcy's law and the definition of the Münch number. This equation is solved by the following expression for the velocity, with $m=\sqrt{M}, \hat{c}=c / c_{0}$, and $s=x / L$ :

$$
\begin{aligned}
u(x)= & \frac{2 L L_{p} R T c_{0}}{r}\left\{\frac{\sinh \left(m \frac{x}{L}\right)}{m \cosh m}\left[1-\frac{p(L)}{R T c_{0}}\right]\right. \\
& +\cosh \left(m \frac{x}{L}\right) \int_{0}^{x / L} \cosh \left(m s^{\prime}\right)\left[\hat{c}\left(s^{\prime}\right)-1\right] d s^{\prime} \\
& +\sinh \left(m \frac{x}{L}\right) \int_{x / L}^{1} \sinh \left(m s^{\prime}\right)\left[\hat{c}\left(s^{\prime}\right)-1\right] d s^{\prime} \\
& \left.-\tanh m \sinh \left(m \frac{x}{L}\right) \int_{0}^{1} \cosh \left(m s^{\prime}\right)\left[\hat{c}\left(s^{\prime}\right)-1\right] d s^{\prime}\right\}
\end{aligned}
$$

as can be verified by differentiating the solution twice and inserting into (A1). Thus, the velocity at the end of the conduit is

$$
\begin{aligned}
u(L)= & \frac{2 L_{p} L R T c_{0}}{r}\left\{\frac{\tanh m}{m}\left[1-\frac{p(L)}{R T c_{0}}\right]\right. \\
& \left.+\frac{1}{\cosh m} \int_{0}^{1} \cosh \left(m s^{\prime}\right)\left[\hat{c}\left(s^{\prime}\right)-1\right] d s^{\prime}\right\} \\
= & u(L)_{c=c_{0}}+\frac{2 L L_{p} R T c_{0}}{r \cosh m} \int_{0}^{1} \cosh \left(m s^{\prime}\right)\left[\hat{c}\left(s^{\prime}\right)-1\right] d s^{\prime} .
\end{aligned}
$$

If $c$ can never exceed $c_{0}$, the integral in (A3) can never give a positive contribution to the output velocity. The maximally achievable velocity is therefore the one found for the constant concentration case, $u(L)_{c=c_{0}}$ [see (9)].
[1] Theophrastus, in Enquiry into Plants, edited by A. F. Hort (Harvard University Press, Cambridge, MA, 1916).

[2] S. Díaz, J. Kattge, J. H. Cornelissen, I. J. Wright, S. Lavorel, S. Dray, B. Reu, M. Kleyer, C. Wirth, I. C. Prentice et al., Nature (London) 529, 167 (2016).

[3] G. Kunstler, D. Falster, D. A. Coomes, F. Hui, R. M. Kooyman, D. C. Laughlin, L. Poorter, M. Vanderwel, G. Vieilledent, S. J. Wright et al., Nature (London) 529, 204 (2016).

[4] Y. Onoda, M. Westoby, P. B. Adler, A. M. Choong, F. J. Clissold, J. H. Cornelissen, S. Díaz, N. J. Dominy, A. Elgart, L. Enrico et al., Ecol. Lett. 14, 301 (2011).

[5] K. J. Niklas, Plant Biomechanics (University of Chicago Press, Chicago, 1992).

[6] K. H. Jensen and M. A. Zwieniecki, Phys. Rev. Lett. 110, 018104 (2013).

[7] M. A. Zwieniecki and C. K. Boyce, Proc. R. Soc. B 281, 20132829 (2014).

[8] K. H. Jensen, J. Liesche, T. Bohr, and A. Schulz, Plant, Cell Environ. 35, 1065 (2012).

[9] H. Ronellenfitsch, J. Liesche, K. H. Jensen, N. M. Holbrook, A. Schulz, and E. Katifori, Proc. R. Soc. B 282, 20141863 (2015).

[10] G. W. Koch, S. C. Sillett, G. M. Jennings, and S. D. Davis, Nature (London) 428, 851 (2004).
[11] O. Dorado, G. Avila, D. M. Arias, R. Ramirez, D. Salinas, and G. Valladares, Madroño 43, 445 (1996).

[12] D. R. Currey, Ecology 46, 564 (1965).

[13] R. M. Burns, B. H. Honkala et al., Silvics of North America. Conifers, Agriculture Handbook 654 (US Department of Agriculture, Washington, DC, 1990), Vol. 1, pp. 552-562.

[14] L. Taiz and E. Zeiger, Plant Physiology, 5th ed. (Sinauer Associates, Sunderland, MA, 2010).

[15] C. J. Earle, The Gymnosperm Database. Pinidae, http://www. conifers.org/zz/pinales.htm.

[16] See Supplemental Material at http://link.aps.org/supplemental/ 10.1103/PhysRevE.95.042402 for conifer leaf lengths and source references.

[17] M. V. Thompson and N. M. Holbrook, J. Theor. Biol. 220, 419 (2003).

[18] W. F. Pickard and B. Abraham-Shrauner, Func. Plant Biol. 36, 629 (2009).

[19] A. D. Stroock, V. V. Pagay, M. A. Zwieniecki, and N. M. Holbrook, Annu. Rev. Fluid Mech. 46, 615 (2014).

[20] K. Jensen, K. Berg-Sørensen, H. Bruus, N. Holbrook, J. Liesche, A. Schulz, M. Zwieniecki, and T. Bohr, Rev. Mod. Phys. 88, 035007 (2016).

[21] J. Landsberg and N. Fowkes, Ann. Bot. 42, 493 (1978). 
[22] M. A. Zwieniecki, H. A. Stone, A. Leigh, C. K. Boyce, and N. M. Holbrook, Plant, Cell Environ. 29, 803 (2006).

[23] L. A. Segel, J. Theor. Biol. 29, 233 (1970).

[24] C. Lin and L. A. Segel, Mathematics Applied to Deterministic Problems (SIAM, Philadelphia, 1974) .

[25] E. Münch, Die Stoffbewegungen in der Pflanze (Gustav Fischer, Jena, 1930).

[26] Ronellenfitsch et al. [9] found that $50 \%$ of conduits were longer than $75 \%$ of the needle length.

[27] This expression is valid for a cylindrical cell of radius $r$; in other geometries the factor $2 / r$ should be replaced by the relevant cell surface-to-volume ratio.

[28] J. M. Dannenhoffer, W. Ebert, Jr., and R. F. Evert, Am. J. Bot. 77, 636 (1990).

[29] S. Russell and R. F. Evert, Planta 164, 448 (1985).

[30] K. H. Jensen, D. L. Mullendore, N. M. Holbrook, T. Bohr, M. Knoblauch, and H. Bruus, Front. Plant Sci. 3, 1 (2012).

[31] E. A. Rennie and R. Turgeon, Proc. Natl. Acad. Sci. USA 106, 14162 (2009).

[32] J. Liesche, H. J. Martens, and A. Schulz, Protoplasma 248, 181 (2011).
[33] K. H. Jensen, J. Lee, T. Bohr, H. Bruus, N. M. Holbrook, and M. A. Zwieniecki, Interface 8, 1155 (2011).

[34] H. Rademaker, K. H. Jensen, and T. Bohr, arXiv:1610.09175 (2016).

[35] A. B. Leslie, J. M. Beaulieu, H. S. Rai, P. R. Crane, M. J. Donoghue, and S. Mathews, Proc. Natl. Acad. Sci. USA 109, 16217 (2012).

[36] S. T. Gower and J. M. Norman, Ecology 72, 1896 (1991).

[37] H. Poorter, K. J. Niklas, P. B. Reich, J. Oleksyn, P. Poot, and L. Mommer, New Phytol. 193, 30 (2012).

[38] C. Brouat, M. Gibernau, L. Amsellem, and D. McKey, New Phytol. 139, 459 (1998).

[39] C. Stromberg, Annu. Rev. Earth Planet. Sci. 39, 517 (2011).

[40] K. H. Jensen, J. A. Savage, and N. M. Holbrook, Interface 10, 20130055 (2013).

[41] D. B. Fisher and J. M. Frame, Planta 161, 385 (1984).

[42] S. Long, P. Farage, and R. Garcia, J. Exp. Bot. 47, 1629 (1996).

[43] A. Graf, A. Schlereth, M. Stitt, and A. M. Smith, Proc. Natl. Acad. Sci. USA 107, 9458 (2010).

[44] K. J. Niklas, Plant allometry: the Scaling of Plant Form and Process (University of Chicago Press, Chicago, 1994).

[45] R. Turgeon, Plant Physiol. 152, 1817 (2010). 